\title{
Molecular epidemiology of sequence type 33 of Shiga toxin-producing Escherichia coli 091:H14 isolates from human patients and retail meats in Korea
}

\author{
Jun Bong Lee ${ }^{1}$, Se-Kye Kim ${ }^{1}$, Seon Mi Wi ${ }^{1}$, Young-Jae Cho ${ }^{1}$, Tae-Wook Hahn ${ }^{1}$, Jae-yon Yu $^{2}$, Sungsun Kim ${ }^{2}$, Sahyun Hong ${ }^{3}$, \\ Jonghyun $\mathrm{Kim}^{2, *}$, Jang Won Yoon ${ }^{1, *}$ \\ ${ }^{I}$ College of Veterinary Medicine and Institute of Veterinary Science, Kangwon National University, Chuncheon 24341, Korea \\ ${ }^{2}$ Division of Bacterial Disease Research, Center for Infectious Diseases Research, Korea National Institute of Health, Centers for Disease Control and \\ Prevention, Cheongju 28159, Korea \\ ${ }^{3}$ Division of Bacterial Disease, Center for Laboratory Control of Infectious Diseases, Center for Disease Control and Prevention, Cheongju 28159, Korea
}

\begin{abstract}
Sequence type (ST) 33 of Shiga toxin-producing Escherichia coli (STEC) strain O91:H14 has been proposed as a potential domestic clone of STEC in Korea because of its high prevalence among human patients with mild diarrhea or asymptomatic carriers. Herein, the clonal diversity of 17 STEC O91:H14 isolates of ST33 during 2003 to 2014 was analyzed by pulsed-field gel electrophoresis, including 14 isolates from human patients and 3 from retail meats. Their virulence characteristics, acid resistance, and antimicrobial susceptibility were also determined. Our results showed that all isolates were clustered mainly into three different pulsotypes and were likely low pathogenic without antimicrobial resistance.
\end{abstract}

Keywords: Shiga toxin; Escherichia coli; Multilocus sequence type; O91; Korea

Since its first outbreak in 1982, Shiga toxin-producing Escherichia coli (STEC) has caused many illnesses in humans, including hemorrhagic colitis (HC), hemolytic uremic syndrome (HUS), and thrombotic thrombocytopenic purpura [13]. Although E. coli strains of all known O serogroups appear to be associated with the production of Shiga toxins (Stxs), the single serovar O157:H7 (hereafter referred to as O157 STEC) is known to be responsible for many severe epidemic outbreaks in humans.

In addition to $\mathrm{O} 157 \mathrm{STEC}$, epidemiological surveillance data suggest that four non-O157 STEC serovars, namely O26, O103, O111, and O145, are frequently associated with human outbreaks worldwide, and they have been designated the "big four" non-O157 STEC [3]. Using multilocus sequence typing, interestingly, a recent study demonstrated that the majority ( $\sim 93.6 \%$ ) of those big four non-O157 STEC isolates can be clustered into unique sequence type complexes (STCs) including STC10, STC20, STC29, and STC32, which implies that they are potentially evolved from some shared ancestors or common lineages of E. coli [5]. For example, approximately
$86.9 \%$ of STEC O26 and O111 isolates were assigned to STC29 comprising 15 sequence types (STs) that included ST16, ST21, and ST29 [5]. Most STEC O103 isolates were assigned to STC20 comprising ST17 and ST386, while STEC O145 isolates were assigned to STC32 comprising ST32 and ST137 [11]. Therefore, it was proposed that highly virulent serovars of non-O157 STEC might share similar phylogenetic lineages.

In our attempt to define a pathogenic clone(s) of STEC in Korea, it was demonstrated that the STEC O91:H14 serovar belonged to the ST33 lineage and was highly prevalent among human patients and retail meat samples collected during 2003 to 2014 [8]. Although the isolates do not belong to the big four non-O157 STEC, the same serotypes have been reported among the most common STEC isolates from diarrheal patients without HUS in Germany [11]. Moreover, it has also been reported that ST33 of STEC O91:H14 is a major causative agent of diarrhea in human patients in Japan [10].

In this study, it was hypothesized that STEC O91:H14 isolates with ST33 are phylogenetically related and might be a 
potential domestic clone of STEC in Korea. To that end, a total of 17 STEC O91:H14 belonging to the ST33 lineage were collected from human patients $(n=14)$ or retail meat samples $(\mathrm{n}=3$ ) during 2003 to 2014 (Table 1) and analyzed using standard pulsed-field gel electrophoresis (PFGE). Their virulence potentials were also evaluated by measuring (i) the ability of $E$. coli to produce one or more Stxs, (ii) the presence of previously characterized STEC virulence genes including Stx subtypes, (iii) glutamate-induced acid resistance, and (iv) antimicrobial susceptibility.

As shown in Fig. 1, PFGE profiles with $>80 \%$ similarity were analyzed as previously described [8]. The results showed

Table 1. Prevalence of virulence genes among the 17 STEC O91:H14 isolates belonging to ST33 and isolated in Korea during 2003 to 2014

\begin{tabular}{|c|c|c|c|c|c|c|c|}
\hline \multirow{2}{*}{ STEC isolates (No. of isolates) } & \multirow{2}{*}{ Source (sampling year) ${ }^{*}$} & \multicolumn{6}{|c|}{ Presence of the following virulence genes } \\
\hline & & $\operatorname{sub} A B$ & eae & saa & iha & $e h x A$ & tox $B$ \\
\hline H2003 (1) & Diarrheal patient (2003) & + & - & + & + & + & - \\
\hline $\mathrm{H} 2004(1)$ & Asymptomatic carrier (2004) & + & - & + & + & - & - \\
\hline $\mathrm{H} 2007$ (1) & Diarrheal patient (2007) & + & - & + & + & + & - \\
\hline B2007-75 (1) & Retail beef (2007) & + & - & + & + & + & - \\
\hline B2007-76 (1) & Retail beef (2007) & + & - & + & + & + & - \\
\hline P2010-29 (1) & Retail pork (2010) & - & - & - & - & + & - \\
\hline $\mathrm{H} 2012-1$ to $-4(4)$ & Diarrheal patients (2012) & + & - & + & + & + & - \\
\hline $\mathrm{H} 2013-1$ to $-6(6)$ & Diarrheal patients (2013) & + & - & + & + & + & - \\
\hline $\mathrm{H} 2014(1)$ & Diarrheal patient (2014) & + & - & + & + & + & - \\
\hline Prevalence (positive/total) & & $16 / 17$ & $0 / 17$ & $16 / 17$ & $16 / 17$ & $16 / 17$ & $0 / 17$ \\
\hline
\end{tabular}

STEC, Shiga toxin-producing Escherichia coli; ST, sequence type; subAB, subtilase cytotoxin; eae, intimin; saa, STEC agglutinating adhesin; iha, iron-regulated gene $\mathrm{A}$ homolog adhesin; ehxA, enterohemorrhagic $E$. coli hemolysin; toxB, E. coli putative adhesin encoded in pO157. ${ }^{*}$ Diarrhea represents watery diarrhea without hemorrhage.

\begin{tabular}{|c|c|c|c|c|c|c|c|c|c|c|c|c|c|c|}
\hline \multirow{2}{*}{ ㅇ 8 8 둔 } & \multirow[b]{2}{*}{ PFGE (Xbal) } & \multirow{2}{*}{\multicolumn{2}{|c|}{$\begin{array}{l}\text { STEC Pulsotype } \\
\text { isolates }\end{array}$}} & \multicolumn{2}{|c|}{ stx genotype } & \multicolumn{2}{|c|}{ RPLA titer } & \multirow{2}{*}{$\begin{array}{l}\text { Survival rates (\%) } \\
\text { in AR2 system }\end{array}$} & \multicolumn{6}{|c|}{ Antibiotic resistance } \\
\hline & & & & $s t x_{1}$ & $s t x_{2}$ & stx1 & stx2 & & AM & $\overline{A N}$ & $\mathrm{CF}$ & $\mathrm{CIP}$ & & $\mathrm{TX}$ \\
\hline & 111 & H2013-1 & & $s t x_{1}$ & $s t x_{2 \text { dact }}$ & $1: 8$ & $1: 2$ & $35.1 \pm 11.5^{\star * *}$ & - & - & I & - & 1 & I \\
\hline & 111 & $\mathrm{H} 2013-2$ & & $s t x_{1}$ & $s t x_{2 \text { dact }}$ & $1: 8$ & $1: 2$ & $55.4 \pm 17.7^{\star \star}$ & I & - & I & - & । & - \\
\hline & iil i & H2013-3 & & $s t x_{1}$ & $s t x_{2 \text { dact }}$ & $1: 8$ & $1: 2$ & $52.0 \pm 19.1^{* *}$ & । & - & I & - & । & - \\
\hline & ili & H2013-4 & A & $s t x_{1}$ & $s t x_{2 \text { dact }}$ & $1: 4$ & $1: 2$ & $51.5 \pm 8.9^{* *}$ & 1 & - & 1 & - & I & - \\
\hline & iil & H2013-6 & & $s t x_{1}$ & $s t x_{2 \text { dact }}$ & $1: 4$ & $1: 2$ & $50.4 \pm 5.1^{* *}$ & I & 1 & 1 & - & I & - \\
\hline & IIIi\|. & $\mathrm{H} 2004$ & & $s t x_{1}$ & $s t x_{2 \text { dact }}$ & $1: 32$ & $1: 8$ & $44.8 \pm 9.5^{\star \star \star}$ & 1 & - & 1 & - & I & - \\
\hline & & H2012-1 & & & $s t x_{2 \text { dact }}$ & & $1: 16$ & $48.9 \pm 13.5^{\star \star \star}$ & 1 & $\mathrm{I}$ & 1 & & 1 & $\mathrm{I}$ \\
\hline & & H2012-2 & & & $s t x_{2 \text { dact }}$ & & $1: 32$ & $51.4 \pm 6.1^{* *}$ & । & I & - & & - & 1 \\
\hline & & H2012-3 & B & & $s t x_{2 \text { dact }}$ & & $1: 32$ & $53.3 \pm 16.7^{* *}$ & I & I & 1 & & I & I \\
\hline & ||||| & H2012-4 & & & $s t x_{2 \text { dact }}$ & & $1: 32$ & $50.0 \pm 10.8^{* *}$ & 1 & - & 1 & & 1 & I \\
\hline & ||| & $\mathrm{H} 2003$ & & $s t x_{1}$ & $s t x_{2 \text { dact }}$ & $1: 8$ & $1: 32$ & $64.1 \pm 10.9^{\star}$ & 1 & - & 1 & - & 1 & - \\
\hline & \|\|\|\| & H2013-5 & & $s t x_{1}$ & & $1: 4$ & & $63.1 \pm 16.5^{*}$ & I & - & 1 & - & 1 & - \\
\hline & i||li | & H2014 & & $s t x_{1}$ & & $1: 4$ & & $<1.0^{\star \star \star \star}$ & _- & _ & _- & _- & _ & _- \\
\hline & $\|$ & $\mathrm{H} 2007$ & C & $s t x_{1}$ & & $1: 4$ & & $48.8 \pm 16.6^{\star * *}$ & 1 & _- & _- & _- & I & $\mathrm{I}$ \\
\hline & & B2007-75 & & $s t x_{1}$ & $s t x_{2 b}$ & $1: 32$ & $<1: 2$ & $71.7 \pm 8.62^{* *}$ & - & I & - & - & I & - \\
\hline & ii & B2007-76 & & $s t x_{1}$ & $s t x_{2 \mathrm{~b}}$ & $1: 32$ & $<1: 2$ & $79.7 \pm 14.22$ & - & - & - & - & - & - \\
\hline & & P2010-29 & & $s t x_{1}$ & & $1: 64$ & & $45.3 \pm 11.02^{* \star *}$ & - & - & - & & 1 & - \\
\hline
\end{tabular}

Fig. 1. Pulsed-field gel electrophoresis (PFGE) profiles and virulence characteristics of the 17 Shiga toxin-producing Escherichia coli (STEC) O91: $\mathrm{H} 14$ isolates with sequence type 33. The data, including multilocus sequence types, stx genotypes, reverse passive latex agglutination (RPLA) titers of Shiga toxins (Stxs), glutamate-induced acid resistance, and antibiotic resistance phenotypes, were combined and presented with the pulsotypes. The STEC isolates were divided into three pulsotypes (A to C) based on $>80 \%$ similarity of PFGE profiles. The RPLA titers of Stxs and the acid survival rates (AR2) were quantitated and compared with those of EDL933, which produced both $\mathrm{Stx}_{1}$ and $\mathrm{Stx}_{2}(1: 16$ and $\geq 1: 128 \mathrm{RPLA}$ titers, respectively) as well as surviving well in EG media (pH 2.5) with $1.5 \mathrm{mM}$ glutamate. AM, ampicillin; AN, amikacin; CF, cephalothin; CIP, ciprofloxacin; S, streptomycin; CTX, cefotaxime; I, intermediate resistance. ${ }^{*} p<0.05$, significantly different from EDL933; ${ }^{*} p<0.01$, significantly different from EDL933; $* * * p<0.001$, significantly different from EDL933. 
that 16 of the 17 STEC O91:H14 isolates were classified mainly into three pulsotypes: the first included six isolates from human patients, which possessed both $s t x_{1}$ and $s t x_{2 \text { dact }}$ genes (designated pulsotype A); the second contained five isolates from human patients, which harbored only the $s t x_{2 \text { dact }}$ gene, except one patient (H2003) who had both $s t x_{1}$ and $s t x_{2 \text { dact }}$ genes (pulsotype B); and the third comprised three isolates from human patients that had only the $s t x_{1}$ gene and two from retail beef samples harboring both $s t x_{1}$ and $s t x_{2 \mathrm{~b}}$ genes (pulsotype C). One isolate from pork (P2010-29) containing only the $s t x_{1}$ gene was included as an outgroup with a distinct PFGE profile from the others (Fig. 1).

Heterogeneity of PFGE patterns and stx genotypes within the ST33 lineage of STEC O91:H14 was previously observed in Japan [10]. Indeed, the 26 ST33 of STEC O91 isolates from human patients in Japan were assigned to two different PFGE clusters [10]. The first cluster contained sixteen isolates, which had only the $s t x_{1}$ gene whereas the second had ten isolates that possessed both $s t x_{1}$ and $s t x_{2}$ (or $s t x_{2 \text { dat }}$ ) genes [10]. In Netherlands, the nineteen ST33 of STEC O91:H14 isolates carried diverse Stx subtype genes $\left(s t x_{1}, s t x_{2}\right.$, and $\left.s t x_{1}+s t x_{2 b}\right)$ [6]. Based on these results, we speculate that ST33 of STEC O91:H14 has evolved in different ways in different countries and their genetic divergence may include variation of $s t x$ genotypes.

In general, STEC strains with the $s t x_{2}$ gene are considered to cause more severe diseases than those carrying $s t x_{1}$. Especially, Stx ${ }_{2 \text { dact, }}$, which was identified in our pulsotype A and B clusters, was reported to be as toxic as $s t x_{2}$ in a murine infection model [9]. Therefore, the production of Stxs was quantitated by using a commercially available reverse passive latex agglutination (RPLA) kit according to the manufacturer's instruction (VTECRPLA; Denka Seiken, Japan). As shown in Fig. 1, however, the RPLA assays revealed that all six STEC isolates belonging to the pulsotype A produced notably lower levels of $\mathrm{Stx}_{2}(1: 2-1: 8)$ than that from EDL933, an O157 STEC isolate from a human outbreak in the USA ( $\geq 1: 128)$. Likewise, the five isolates belonging to pulsotype $\mathrm{B}$ expressed low levels of $\mathrm{Stx}_{2}$ (1:16$1: 32$ ), whereas the two isolates belonging to pulsotype $\mathrm{C}$ produced undetectable levels of $\operatorname{Stx}_{2 b}(<1: 2)$.

As determined via polymerase chain reaction analyses, all 17 ST33 of STEC O91:H14 isolates lacked the eae gene encoding intimin at the locus of enterocyte effacement pathogenicity island, which is required for full virulence of STEC (Table 1). Instead of missing the eae gene, they carried one or more alternative virulence genes encoding SubAB (subtilase cytotoxin $\mathrm{AB}$ ), Saa (STEC autoagglutinating adhesion), Iha (IgrA homolog adhesion), and EhxA (enterohemolysin A) $[4,10]$ (Table 1). Interestingly, a significant reduction in acid resistance phenotypes, compared to EDL933, was observed in most STEC isolates, except B2007-76 (Fig. 1); however, the reduction's biological significance in vivo was not evaluated. In this study, the glutamate-inducible acid resistance of STEC isolates was evaluated using EG media containing $1.5 \mathrm{mM}$ glutamate ( $\mathrm{pH} 2.5$ ) as previously described [8].

Taken together, these results indicate that our 17 ST33 of STEC 091:H14 isolates might have low pathogenicity and be ineffective in causing severe diseases such as HC and HUS in humans. Supporting this notion, the 14 human isolates were obtained from asymptomatic carriers and/or patients with mild watery diarrhea (Table 1). Moreover, a close phylogenetic relationship between the STEC O91 isolates with ST33 lineage and their low pathogenic potentials have been reported in other countries. In Europe, for example, STEC O91 has been the most common O serovar of the eae-negative STEC isolates $[1,12,14,15]$. Among them, O91:H14 and O91:H21 were the dominant serotypes in human patients, and they were divided into two distinct ST lineages; ST33 and ST442 [1,11]. Although most ST33 of STEC O91:H14 isolates seem to be associated with mild diseases [2,6,10,11], ST442 of STEC O91:H21 isolates have been epidemiologically linked to more severe diseases such as HC and HUS [2,7,11].

It is noteworthy that our STEC O91:H14 isolates appear to have low resistance to the antimicrobials tested in this study. Using a standard disk diffusion method with interpretive criteria of the inhibitory zone diameters $(\mathrm{mm})$ according to Clinical and Laboratory Standard Institute standards, we observed that all strains were susceptible or intermediate resistant to one or more antimicrobials, including ampicillin (10 $\mu \mathrm{g}$; BD, USA), amikacin (30 $\mu \mathrm{g}$; BD), cephalothin (30 $\mu \mathrm{g}$; BD), ciprofloxacin $(5 \mu \mathrm{g} ; \mathrm{BD})$, streptomycin $(10 \mu \mathrm{g} ; \mathrm{BD})$, and cefotaxime (30 $\mu$; BD) (Fig. 1).

In conclusion, we characterized the ST33 of STEC O91:H14 isolates, a potential domestic clone of STEC in Korea. Our results imply that a low-pathogenic clone of STEC O91:H14 isolates with ST33 lineage is likely present and has divergently evolved in Korea.

\section{Acknowledgments}

This study was supported by a grant (No. Z-15417782013-14-02) from Animal and Plant Quarantine Agency, Ministry of Agriculture, Food, and Rural Affairs, Republic of Korea, and by Research of Korea Centers for Disease Control and Prevention (No. 4800-4851-304).

\section{Conflict of Interest}

The authors declare no conflicts of interest.

\section{References}

1. Bettelheim KA. The non-O157 Shiga-Toxigenic (Verocytotoxigenic) Escherichia coli; under-rated pathogens. 
Crit Rev Microbiol 2007, 33, 67-87.

2. Bielaszewska M, Stoewe F, Fruth A, Zhang W, Prager R, Brockmeyer J, Mellmann A, Karch H, Friedrich AW. Shiga toxin, cytolethal distending toxin, and hemolysin repertoires in clinical Escherichia coli O91 isolates. J Clin Microbiol 2009, 47, 2061-2066.

3. Brooks JT, Sowers EG, Wells JG, Greene KD, Griffin PM, Hoekstra RM, Strockbine NA. Non-O157 Shiga toxinproducing Escherichia coli infections in the United States, 1983-2002. J Infect Dis 2005, 192, 1422-1429.

4. Cergole-Novella MC, Nishimura LS, Dos Santos LF, Irino K, Vaz TM, Bergamini AM, Guth BE. Distribution of virulence profiles related to new toxins and putative adhesins in Shiga toxin-producing Escherichia coli isolated from diverse sources in Brazil. FEMS Microbiol Lett 2007, 274, 329-334.

5. Eichhom I, Heidemanns K, Semmler T, Kinnemann B, Mellmann A, Harmsen D, Anjum MF, Schmidt H, Fruth A, Valentin-Weigand P, Heesemann J, Suerbaum S, Karch H, Wieler LH. Highly virulent Non-O157 enterohemorrhagic Escherichia coli (EHEC) serotypes reflect similar phylogenetic lineages, providing new insights into the evolution of EHEC. Appl Environ Microbiol 2015, 81, 7041-7047.

6. Ferdous M, Friedrich AW, Grundmann H, de Boer RF, Croughs PD, Islam MA, Kluytmans-van den Bergh MF, Kooistra-Smid AM, Rossen JW. Molecular characterization and phylogeny of Shiga toxin-producing Escherichia coli isolates obtained from two Dutch regions using whole genome sequencing. Clin Microbiol Infect 2016, 22, 642.e1-9.

7. Hauser E, Mellmann A, Semmler T, Stoeber H, Wieler LH, Karch H, Kuebler N, Fruth A, Harmsen D, WenigerT, Tietze E, Schmidt H. Phylogenetic and molecular analysis of food-borne shiga toxin-producing Escherichia coli. Appl Environ Microbiol 2013, 79, 2731-2740.

8. Lee JB, Han D, Lee HT, Wi SM, Park JH, Jo JW, Cho YJ,
Hahn TW, Lee S, Kang B, Kwak HS, Kim J, Yoon JW. Pathogenic and phylogenetic characteristics of non-O157 Shiga toxin-producing Escherichia coli isolates from retail meats in South Korea. J Vet Sci 2018, 19, 251-259.

9. Lindgren SW, Samuel JE, Schmitt CK, O'Brien AD. The specific activities of Shiga-like toxin type II (SLT-II) and SLT-II-related toxins of enterohemorrhagic Escherichia coli differ when measured by Vero cell cytotoxicity but not by mouse lethality. Infect Immun 1994, 62, 623-631.

10. Maeda E, Murakami K, Etoh Y, Onozuka D, Sera N, Asoshima N, Honda M, Narimatsu H, Iyoda S, Watahiki M, Fujimoto S. Does sequence type 33 of Shiga toxin-producing Escherichia coli O91 cause only mild symptoms? J Clin Microbiol 2015, 53, 362-364.

11. Mellmann A, Fruth A, Friedrich AW, Wieler LH, Harmsen D, Werber D, Middendorf B, Bielaszewska M, Karch $\mathbf{H}$. Phylogeny and disease association of Shiga toxin-producing Escherichia coli O91. Emerg Infect Dis 2009, 15, 14741477.

12. Merkel V, Ohder B, Bielaszewska M, Zhang W, Fruth A, Menge C, Bormann E, Middendorf B, Müthing J, Karch H, Mellmann A. Distribution and phylogeny of immunoglobulinbinding protein $\mathrm{G}$ in Shiga toxin-producing Escherichia coli and its association with adherence phenotypes. Infect Immun 2010, 78, 3625-3636.

13. Nataro JP, Kaper JB. Diarrheagenic Escherichia coli. Clin Microbiol Rev 1998, 11, 142-201.

14. Werber D, Behnke SC, Fruth A, Merte R, Menzler S, Glaser S, Kreienbrock L, Prager R, Tschäpe H, Roggentin P, Bockemühl J, Ammon A. Shiga toxin-producing Escherichia coli infection in Germany: different risk factors for different age groups. Am J Epidemiol 2007, 165, 425-434.

15. Werber D, Beutin L, Pichner R, Stark K, Fruth A. Shiga toxin-producing Escherichia coli serogroups in food and patients, Germany. Emerg Infect Dis 2008, 14, 1803-1806. 